\title{
Non Parametric Analysis in Multi Environmental Trials of Feed Barley Genotypes
}

\author{
Ajay Verma*, J. Singh, V. Kumar, A.S. Kharab and G.P. Singh \\ Statistics and computer center, ICAR-Indian Institute of Wheat and Barley Research, \\ Karnal 132001 Haryana, India \\ *Corresponding author
}

\section{A B S T R A C T}

Key w or d s
Feed barley, Non-
parametric
measures, Spearman
rank correlation,
Ward's hierarchical
clustering, Biplot
analysis.
Article Info
Accepted:
19 May 2017
Available Online:
10 June 2017

\section{Keywords}

Feed barley, Nonparametric measures, Spearman rank correlation, clustering, Biplot analysis.

10 June 2017
GXE interaction of twenty seven feed barley genotypes tested at fifteen major barley locations interpreted by non-parametric measures. Genotype JB322 was high yielder followed by PL890 and HUB250 among feed barley genotypes. Descriptive statistics calculated from original yield values identified genotypes KB1436 and KB1434 were of stable performance. Nonparametric measures, free from distributional assumptions, Si1, Si2, Si3, Si4, Si5 Si6 and Si7 indicated JB322, UPB1054 and KB1434 as stable genotypes, however, unstable genotypes were RD2552G5 and DWRB156. CMR and CSD measures pointed towards HUB113, NDB1634 and UPB1054, JB322 as desirable genotypes respectively. CSi1 and CSi2 measures identified JB322 and UPB1054 along with UPB1054 and HUB 113 as of stable yield performance. Genotypes UPB1054, HUB113, BH1005 based on CSi3 and CSi6 were identified as the stable genotypes whereas KB1436 and RD2552 were unstable. First two NPs were very similar for unstable performance of RD2552 and last two NPs for similar performance of HUB250.

\section{Introduction}

Barley cereal crop has been cultivated for food, feed, forage and brewing purpose. Cereal is grown under varying agro climatic situations of the country. Interpretation of genotype $\mathrm{x}$ environment interactions facilitated by the use of statistical methods as interaction complicates the identification of superior genotypes (Berteroa et al., 2004). The nonparametric measures, based on ranks only, proved to be a viable alternative to parametric measures (Dehghani, 2008). For many applications, including selection in breeding and testing programs, the rank orders of genotypes are the most essential information (Khalili and Aboughadareh, 2016). Quite large number of references justifies the use of nonparametric measures in the assessment of stable performance in crop improvement trials (Ebadi-Segherloo, 2008; Karimizadeh et al., 2012). Nonparametric measures based rank of genotypes as per the yield values in different environments were developed by Huehn (1990a). The use of corrected values, instead of original means, for rank determination was proposed by Huehn (1990b). Nonparametric measures of phenotypic stability by Huehn have been used numerously (Dehghani, 2008; Ebadi-Segerloo 
et al., 2008; Mahtabi et al., 2013; Liu et al., 2010; Sabaghnia et al., 2006; Karimzadeh et al., 2013). Recent papers have exploited nonparametric measures to analyze $\mathrm{GxE}$ interaction in agricultural trials (Mortazavian and Azizinia, 2014). Nonparametric stability statistics had been used by plant breeders worldwide due to the potential returns relative to other parameters (Farshadfar et al., 2014).

\section{Materials and Methods}

Research data of this study involved twenty seven feed barley genotypes evaluated at fifteen major barley growing locations across the country. Environmental conditions along with genotype pedigrees were given in table 1 for completeness. Xij denotes the phenotypic value of ith genotype in jth environ $\neg$ ment, where $i=1,2, \ldots k, j=1,2, \ldots, n$. Rank of the ith genotype in the jth environment denoted by rij and the average rank of the ith genotype across environments by. (Karimizadeh et al., 2012) Sil estimate considered all possible pair-wise rank differences, while $\mathrm{Si} 2$ was based on variances of ranks for each genotype across environments. The nonparametric stability statistic $\mathrm{Si} 4$ is similar to that of Yau and Hamblin (1994), which used relative yield not only to give equal weight to each environment, but also to provide a measure of yield stability. Non-parametric statistics of $\mathrm{Si} 3$ and Si6 combine yield and stability based on yield ranks of genotypes in each environment. Karimzadeh et al., 2013 proposed the correction for yield of ith genotype in $\mathrm{jth}$ environment as $\left(\mathrm{X}^{*} \mathrm{ij}=\mathrm{Xij}\right.$ .+) as $\mathrm{X}^{*} \mathrm{ij}$, was the corrected phenotypic value;. was the mean of ith genotype in all environments and was the grand mean. The ranks obtained from these adjusted values $\mathrm{X}^{*} \mathrm{ij}$, depend only on $\mathrm{GxE}$ interaction and error effects. The genotype with the highest adjusted yield was given a rank of 1 and vice versa for lowest adjusted yielder. Following nonparametric measures were calculated based on the ranks assigned by original and corrected values of yield to genotypes as:

$$
\begin{array}{ll}
S_{i}^{(1)}=2 \sum_{j}^{n-1} \sum_{j=j+1}^{n}\left|r_{i j}-r_{i j}\right| l[\mathrm{n}(\mathrm{n}-1)] & S_{i}^{(4)}=\sqrt{\frac{\sum_{j=1}^{n}\left(r_{i j}-\bar{r}_{i .}\right)^{2}}{n}} \\
S_{i}^{(2)}=\sum_{j=1}^{n}\left(r_{i j}-\bar{r}_{i}\right)^{2} / \sum_{j=1}^{n}\left|r_{i j}-\bar{r}_{i .}\right| & S_{i}^{(5)}=\frac{\sum_{j=1}^{n}\left|r_{i j}-\bar{r}_{i .}\right|}{n} \\
S_{i}^{(3)}=\frac{\sum_{j=1}^{n}\left(r_{i j}-\bar{r}_{i .}\right)^{2}}{\bar{r}_{i .}} & S_{i}^{(6)}=\frac{\sum_{j=1}^{n}\left|r_{i j}-\bar{r}_{i j}\right|}{\bar{r}_{i .}}
\end{array}
$$

On parametric measures to measure stability defined by Thennarasu's (1995). In these measures $\mathrm{r}^{*} \mathrm{ij}$ was the rank of $\mathrm{X} * \mathrm{ij}$, and and Mdi were the mean and median ranks for original, where $*$ and $\mathbf{M}^{*}$ di were the same parameters computed from the corrected yield values.

$$
\begin{gathered}
\mathrm{NP}_{i}^{(1)}=\frac{1}{m} \sum_{j=1}^{m}\left|r_{i j}^{*}-M_{d i}^{*}\right| \\
\mathrm{NP}_{i}^{(2)}=\frac{1}{m}\left(\sum_{j=1}^{m} \frac{\left|r_{i j}^{*}-M_{d i}^{*}\right|}{M_{d i}}\right) \\
\mathrm{NP}_{i}^{(3)}=\frac{\sqrt{\sum\left(r_{i j}^{*}-\bar{r}_{i .}^{*}\right)^{2} / m}}{\bar{r}_{i .}} \\
\mathrm{NP}_{i}^{(4)}=\frac{2}{m(m-1)}\left[\sum_{j=1}^{m-1} \sum_{j=j+1}^{m} \frac{\left|r_{i j}^{*}-r_{i j}^{*}\right|}{\bar{r}_{i .}}\right]
\end{gathered}
$$

SAS-based computer program of Lu (1995) and SASGESTAB (Hussein et al., 2000) employed to calculate nonparametric measures. Hierarchical clustering of genotypes based on yield along with non parametric measures by Ward's method (Ward, 1963) was performed to understand the relationships among the nonparametric methods. Spearman's rank correlation coefficient calculates the correlation among 
ranks as follows :

$$
r_{s}=1-\frac{6 \sum_{i=1}^{n} d_{i}^{2}}{n\left(n^{2}-1\right)}
$$

$$
\begin{aligned}
& d_{i}=\text { difference between two ranks of investigated trait; } \\
& \mathrm{n}=\text { number of correlated pairs. }
\end{aligned}
$$

\section{Results and Discussion}

According to mean yield, genotype JB322 was the highest yielder followed by PL890 and HUB250, although remarkable differences were evident among the studied feed barley genotypes (Table 2).

The following three descriptive statistics; mean of ranks (MR), standard deviation of ranks (SD) and coefficient of variation of ranks $(\mathrm{CV})$ were calculated for original ranks. According to these statistics, genotypes KB1436 and KB1434 were of stable performance, while genotypes JB322, JB325 and PL890 based on MR, genotypes DWRB156 and RD2552 based on SD and genotypes HUB250 and BH946 based on CV, were identified as of unstable nature. Simple descriptive statistics based on ranks discriminated among genotype performance (Karimzadeh et al., 2012).

Seven nonparametric measures ( $\mathrm{Si} 1, \mathrm{Si} 2, \mathrm{Si}$, $\mathrm{Si} 4, \mathrm{Si} 5 \mathrm{Si} 6$ and $\mathrm{Si} 7$ ) based on original yield values indicated genotypes JB322, UPB1054 and KB1434 were the most stable, however, most of studied measures pointed towards RD2552G5 and DWRB156 as the unstable genotypes, stable genotypes according to Huehn's nonparametric measures from uncorrected values demonstrated high mean yield. In other words, with maintenance of genotype effect in each cell of two-way data, mean yield confounds GEI and affects stability analysis (Farshadfar et al., 2014). Simultaneous selection for both mean yield and stability is an important consideration as
Kang and Pham (1991) studied several stability methods simultaneous for yield and stability. These methods provide a lot of flexibility for plant breeders for the simultaneous selection for both mean yield and stability (Mohammadi et al., 2007).

According to table 3, genotype JB322 followed by UPB1054 were the most stable as well as RD2552 and DWRB156 were of unstable performance based on a corrected dataset that produced a mean of corrected ranks (CMR), standard deviation of corrected ranks (CSD), coefficient of variation of corrected ranks (CCV) and all Huehn's nonparametric measures (CSi1, CSi2, CSi3, CSi4, CSi5 CSi6 and CSi7). Also genotypes UPB1054 and HUB113 were identified as the most stable and KB1436 and RD2552 were unstable based on the above mentioned nonparametric measures of phenotypic stability (Karimizadeh et al., 2012). In the mentioned strategy, the following concept of stability was applied; it determines the stability of genotype over environment if its rank is similar over other environments (biological concept). Many authors (EbadiSegerloo et al., 2008; Zali et al., 2011; Sabaghnia et al., 2006) have used the nonparametric measures based on corrected values for stability and demonstrated that these statistics were associated with the biological concept of stability.

Nonparametric indices of Thennarasu's evaluated the genotypes performance differently i.e. NPi (1) pointed towards JB322 and UPB1054 as stable in comparison to others and RD2552 along BH946 unstable (Table 3) while, genotype PB891 showed lowest value NPi (2) followed by KB1434 and because of high value stabilities of BH946 and RD2552 were low, NPi (3) unlike NPi (2) identified BH1005 as the most stable followed by BH949. The unstable genotypes based on NPi (3) were JB322 and HUB250. Stability parameters NPi (4) like NPi (2) 
identified PB891 and KB1434 and BH946 but like NPi(3) pointed towards unstable performance of HUB250. The results of first two NPs were very similar for unstable performance of RD2552 and last two NPs towards HUB250 as unstable genotypes (Mohammadi et al., 2007).

\section{Clustering of genotypes as per non parametric measures}

Ward's method of hierarchical cluster analysis exploited to group genotypes according to yield and different nonparametric measures of phenotypic stability. The clustering considered squared Euclidean distance as dissimilarity measure among genotypes in Ward's method (Figure 2). In Ward's procedure, the dissimilarity between two clusters is shown by the "loss of information" from joining the two clusters with this loss of information measured by the increase in error sum of squares. The cluster analysis revealed four distinct clusters among twenty seven genotypes: cluster of high to moderate yielders consisted of genotypes JB890, PL890, BH902, and RD2922 as UPB1054 as the most favorable and next cluster of four genotypes consisted of unstable genotypes RD2552, DWRB156, BH946 and HUB250. Third cluster of six genotypes comprised of moderately yielder genotypes. Finally fourth cluster grouped highly unstable genotypes as per non parametric measures. It seems that according to corrected statistics, genotypes UPB1054, HUB113 and JB322 were the most stable, but when based on uncorrected statistics, genotypes UPB1054 and KB1434 were the most stable. Regarding mean yield regardless of stability, the most favorable genotypes were JB322 and PL890.

Table.1 Parentage details of feed barley genotypes along with environmental conditions

\begin{tabular}{|c|c|c|c|c|c|c|}
\hline Code & Genotype & Parentage & Locations & Latitude & Longitude & Altitude (m) \\
\hline IVTIRFB-1 & KB1436 & LAKHAN/JB137 & Durgapura & $26^{\circ} 51 \mathrm{~N}$ & $75^{\circ} 47^{\prime} \mathrm{E}$ & 390 \\
\hline IVTIRFB-2 & BH959 & BH393/BH331 & Hisar & $29^{\circ} 10^{\prime} \mathrm{N}$ & $75^{\circ} 46^{\prime} \mathrm{E}$ & 215.2 \\
\hline IVTIRFB-3 & RD2922 & RD2809/RD2743 & Ludhiana & $30^{\circ} 54^{\prime} \mathrm{N}$ & $75^{\circ} 52^{\prime} \mathrm{E}$ & 247 \\
\hline IVTIRFB-4 & HUB250 & RD2618/RD2660 & Tabiji & $26^{\circ} 35^{\prime} \mathrm{N}$ & $74^{\circ} 61^{\prime} \mathrm{E}$ & 456.1 \\
\hline IVTIRFB-5 & BH1004 & 33rd IBON200/BH902 & Pant Nagar & $29^{\circ} 02^{\prime} \mathrm{N}$ & $79^{\circ} 48^{\prime} \mathrm{E}$ & 237 \\
\hline IVTIRFB-6 & UPB1054 & IBYT-LRA-M-12(Sr.No.27 of EIBGN 2013-14) & Karnal & $29^{\circ} 43^{\prime} \mathrm{N}$ & $76^{\circ} 58^{\prime} \mathrm{E}$ & 252 \\
\hline IVTIRFB-7 & PL890 & DWRUB52/DWRUB62 & Varanasi & $25^{\circ} 20^{\prime} \mathrm{N}$ & $83^{\circ} 03^{\prime} \mathrm{E}$ & 75.5 \\
\hline IVTIRFB-8 & JB325 & RD2615/DL88 & Rewa & $24^{\circ} 31^{\prime} \mathrm{N}$ & $81^{\circ} 15^{\prime} \mathrm{E}$ & 365.7 \\
\hline IVTIRFB-9 & BH1006 & 15th HBSN-4/BH902 & Faizabad & $26^{\circ} 47^{\prime} \mathrm{N}$ & $82^{\circ} 12^{\prime} \mathrm{E}$ & 113 \\
\hline IVTIRFB-10 & HUB113 & KARAN280/C138 & Kanpur & $26^{\circ} 29^{\prime} \mathrm{N}$ & $80^{\circ} 18^{\prime} \mathrm{E}$ & 125.9 \\
\hline IVTIRFB-11 & KB1434 & $\begin{array}{l}\text { GLORIA- } \\
\text { BAR/COPAL//PM5/BEN/3/SEN/4/PETUNIA1/5/BBSC/CONGON } \\
\text { A// BLLU/3/CIRU }\end{array}$ & Sabour & $25^{\circ} 24^{\prime} \mathrm{N}$ & $87^{\circ} 04^{\prime} \mathrm{E}$ & 41 \\
\hline IVTIRFB-12 & RD2786 & RD2634/NDB1020//K425 & SK Nagar & $24^{\circ} 19^{\prime} \mathrm{N}$ & $72^{\circ} 19^{\prime} \mathrm{E}$ & 154.5 \\
\hline IVTIRFB-13 & BH902 & BH495/RD2552 & Sagar & $23^{\circ} 83^{\prime} \mathrm{N}$ & $78^{\circ} 73^{\prime} \mathrm{E}$ & 523 \\
\hline IVTIRFB-14 & JB322 & JB101/BH331 & Morena & $26^{\circ} 56^{\prime} \mathrm{N}$ & $78^{\circ} 80^{\prime} \mathrm{E}$ & 152 \\
\hline IVTIRFB-15 & UPB1053 & IBYT-MRA-12(Sr.No.35 of EIBGN 2013-14) & Udaipur & $24^{\circ} 34^{\prime} \mathrm{N}$ & $70^{\circ} 42^{\prime} \mathrm{E}$ & 582 \\
\hline IVTIRFB-16 & PB891 & IBON 343/12th HSBN-176 & & & & \\
\hline IVTIRFB-17 & BH1005 & BHMS24A/WG127 & & & & \\
\hline IVTIRFB-18 & HUB249 & RD2618/RD2660 & & & & \\
\hline IVTIRFB-19 & NDB1634 & IBON-HI-40 (2009-10 & & & & \\
\hline IVTIRFB-20 & BH946 & BHMS22A/BH549//RD2552 & & & & \\
\hline IVTIRFB-21 & RD2923 & RD2552/RD2786 & & & & \\
\hline IVTIRFB-22 & KB1425 & K508/NDB1295 & & & & \\
\hline IVTIRFB-23 & DWRB157 & $\begin{array}{l}\text { ALANDA02/4/ARIZONA5908/ATHS//ASSE/3/F208.74/5/ALAND } \\
\text { A/3/CI08887/CI05761//LIGNEE640-34 }\end{array}$ & & & & \\
\hline IVTIRFB-24 & RD2921 & RD2508/RD2743 & & & & \\
\hline IVTIRFB-25 & JB319 & LAKHAN/BH353 & & & & \\
\hline IVTIRFB-26 & RD2552 & RD2035/DL472 & & & & \\
\hline IVTIRFB-27 & DWRB156 & $\begin{array}{l}\text { P.STO/3/LBIRAN/UNA80//LIGNEE640/4/BLLU/5/PETUNIA } \\
\text { 1/6/M9846//CCXX14.ARZ3/PA }\end{array}$ & & & & \\
\hline
\end{tabular}


Table.2 Descriptive statistics and non-parametric measures based on original values

\begin{tabular}{|c|c|c|c|c|c|c|c|c|c|c|c|c|c|}
\hline Code & Genotype & Yield (q/ha) & MR & SD & $\mathrm{CV}$ & Med & $\mathrm{S}_{\mathrm{i}}{ }^{1}$ & $\mathrm{~S}_{\mathrm{i}}{ }^{2}$ & $\mathrm{~S}_{\mathrm{i}}{ }^{3}$ & $\mathrm{~S}_{\mathrm{i}}^{4}$ & $\mathrm{~S}_{\mathrm{i}}^{5}$ & $\mathrm{~S}_{\mathrm{i}}{ }^{6}$ & $\mathrm{~S}_{\mathrm{i}}^{7}$ \\
\hline IVTIRFB-1 & KB1436 & 32.52 & 20.87 & 6.13 & 0.29 & 23.00 & 6.82 & 7.26 & 25.19 & 5.84 & 4.83 & 3.47 & 37.55 \\
\hline IVTIRFB-2 & BH959 & 36.74 & 16.67 & 6.43 & 0.39 & 18.00 & 7.52 & 7.24 & 34.76 & 6.06 & 5.33 & 4.80 & 41.38 \\
\hline IVTIRFB-3 & RD2922 & 39.68 & 11.00 & 6.60 & 0.60 & 10.00 & 7.64 & 7.63 & 55.45 & 6.29 & 5.33 & 7.27 & 43.57 \\
\hline IVTIRFB-4 & HUB250 & 41.30 & 10.53 & 8.35 & 0.79 & 9.00 & 9.71 & 9.16 & 92.63 & 6.85 & 7.10 & 10.11 & 69.70 \\
\hline IVTIRFB-5 & BH1004 & 35.55 & 16.87 & 7.14 & 0.42 & 19.00 & 8.29 & 8.07 & 42.32 & 6.83 & 5.89 & 5.24 & 50.98 \\
\hline IVTIRFB-6 & UPB1054 & 39.91 & 11.53 & 5.30 & 0.46 & 13.00 & 6.17 & 5.93 & 34.14 & 4.84 & 4.43 & 5.76 & 28.12 \\
\hline IVTIRFB-7 & PL890 & 41.45 & 10.07 & 6.80 & 0.68 & 7.00 & 7.41 & 8.38 & 64.26 & 5.93 & 5.15 & 7.67 & 46.21 \\
\hline IVTIRFB-8 & JB325 & 40.76 & 9.87 & 6.28 & 0.64 & 10.00 & 7.33 & 7.44 & 55.92 & 6.06 & 4.94 & 7.51 & 39.41 \\
\hline IVTIRFB-9 & BH1006 & 35.52 & 18.40 & 6.66 & 0.36 & 20.00 & 7.77 & 7.44 & 33.78 & 6.44 & 5.57 & 4.54 & 44.40 \\
\hline IVTIRFB-10 & HUB113 & 39.51 & 11.60 & 6.56 & 0.57 & 11.00 & 7.54 & 7.75 & 51.86 & 6.30 & 5.17 & 6.69 & 42.97 \\
\hline IVTIRFB-11 & KB1434 & 33.79 & 19.40 & 6.32 & 0.33 & 20.00 & 7.20 & 7.48 & 28.85 & 6.11 & 4.99 & 3.86 & 39.97 \\
\hline IVTIRFB-12 & RD2786 & 40.52 & 11.93 & 7.89 & 0.66 & 13.00 & 9.22 & 8.96 & 72.98 & 7.26 & 6.48 & 8.15 & 62.21 \\
\hline IVTIRFB-13 & BH902 & 40.99 & 10.13 & 6.59 & 0.65 & 8.00 & 7.45 & 7.35 & 59.97 & 6.01 & 5.51 & 8.16 & 43.41 \\
\hline IVTIRFB-14 & JB322 & 41.85 & 8.87 & 4.79 & 0.54 & 9.00 & 5.66 & 5.53 & 36.29 & 4.60 & 3.88 & 6.56 & 22.98 \\
\hline IVTIRFB-15 & UPB1053 & 38.97 & 13.00 & 7.73 & 0.59 & 13.00 & 8.99 & 9.50 & 64.31 & 6.90 & 5.87 & 6.77 & 59.71 \\
\hline IVTIRFB-16 & PB891 & 33.77 & 19.60 & 8.19 & 0.42 & 24.00 & 9.07 & 9.10 & 47.94 & 7.74 & 6.88 & 5.27 & 67.11 \\
\hline IVTIRFB-17 & BH1005 & 37.35 & 15.67 & 6.07 & 0.39 & 15.00 & 7.16 & 6.72 & 32.89 & 5.52 & 5.11 & 4.89 & 36.81 \\
\hline IVTIRFB-18 & HUB249 & 34.93 & 18.00 & 7.65 & 0.43 & 18.00 & 8.91 & 8.72 & 45.56 & 7.23 & 6.27 & 5.22 & 58.57 \\
\hline IVTIRFB-19 & NDB1634 & 37.52 & 14.27 & 7.56 & 0.53 & 13.00 & 8.95 & 8.15 & 56.14 & 6.76 & 6.55 & 6.89 & 57.21 \\
\hline IVTIRFB-20 & BH946 & 41.24 & 11.80 & 8.82 & 0.75 & 9.00 & 10.25 & 9.35 & 92.24 & 8.39 & 7.76 & 9.86 & 77.74 \\
\hline IVTIRFB-21 & RD2923 & 39.59 & 12.73 & 6.86 & 0.54 & 14.00 & 8.11 & 7.82 & 51.75 & 5.89 & 5.62 & 6.62 & 47.07 \\
\hline IVTIRFB-22 & KB1425 & 36.77 & 15.73 & 7.52 & 0.48 & 18.00 & 8.67 & 8.30 & 50.27 & 6.60 & 6.36 & 6.06 & 56.50 \\
\hline IVTIRFB-23 & DWRB157 & 39.57 & 13.93 & 8.17 & 0.59 & 15.00 & 9.56 & 8.89 & 67.10 & 7.89 & 7.01 & 7.55 & 66.78 \\
\hline IVTIRFB-24 & RD2921 & 38.33 & 14.73 & 8.28 & 0.56 & 14.00 & 9.79 & 8.90 & 65.09 & 7.99 & 7.18 & 7.31 & 68.50 \\
\hline IVTIRFB-25 & JB319 & 38.72 & 13.53 & 7.42 & 0.55 & 15.00 & 8.78 & 8.08 & 57.02 & 6.74 & 6.36 & 7.05 & 55.12 \\
\hline IVTIRFB-26 & RD2552 & 39.01 & 13.27 & 9.71 & 0.73 & 11.00 & 11.33 & 10.12 & 99.42 & 9.34 & 8.68 & 9.82 & 94.21 \\
\hline IVTIRFB-27 & DWRB156 & 40.54 & 13.00 & 9.51 & 0.73 & 14.00 & 11.18 & 10.38 & 97.38 & 9.06 & 8.13 & 9.38 & 90.43 \\
\hline
\end{tabular}

Table.3 Descriptive statistics and non-parametric measures based on corrected values

\begin{tabular}{|c|c|c|c|c|c|c|c|c|c|c|c|c|c|c|c|c|}
\hline Code & renotype & CMR & $\mathrm{CSD}$ & $\mathrm{CCV}$ & CMed & CSil & $\mathrm{CSi} 2$ & CSi3 & CSi4 & CSi5 & CSi6 & CSi7 & $\mathrm{NP}_{\mathrm{i}}^{\left({ }^{(1)}\right.}$ & $\mathrm{NP}_{\mathrm{i}}{ }^{(2)}$ & $\mathrm{NP}_{\mathrm{i}}{ }^{(3)}$ & $\mathrm{NP}_{\mathrm{i}}{ }^{(4)}$ \\
\hline IVTIRFB-1 & & 13.33 & 8.99 & 0.67 & 11.00 & 10.53 & 13.93 & 148.70 & 10.53 & 9.49 & 10.68 & 141.61 & 7.667 & 0.333 & 0.551 & 0.505 \\
\hline & & & & 0.51 & 3.00 & & 8.44 & 58.60 & .33 & 6.51 & & & 6.267 & 0.348 & & .506 \\
\hline & & & & 51 & & & 8.83 & & & 5.33 & & & 5.333 & 0.533 & & .713 \\
\hline & & 60 & 87 & 0.61 & 3.00 & & 11.03 & 2.44 & 48 & 8.16 & & & 7.333 & 0.815 & & .991 \\
\hline & & .60 & & 0.63 & 4.00 & & 10.45 & 8.06 & 8.48 & 7.64 & .43 & & 7.333 & 0.386 & 530 & 0.605 \\
\hline & & .33 & 77 & 0.40 & 5.00 & 80 & 7.17 & 0.67 & 6.20 & 5.42 & 5.67 & 41.64 & 4.733 & 0.364 & .541 & 0.590 \\
\hline & & 00 & 81 & 49 & 3.00 & 89 & 9.84 & 3.00 & 6.47 & 5.98 & 5.40 & 63.00 & 5.267 & 0.752 & .762 & 0.783 \\
\hline & & & 65 & 0. & .00 & 71 & 9.22 & 2.00 & 7.50 & 6.21 & 5.72 & & 4.933 & 0.493 & 767 & 0.782 \\
\hline & & 80 & 64 & 0.58 & 7.00 & 9 & 10.83 & 3.70 & 9.05 & 7.63 & 7.73 & 88.49 & 7.533 & 0.377 & .494 & 0.541 \\
\hline-10 & & 40 & 54 & & 2.00 & כ & 7.97 & 9.13 & 6.22 & 5.09 & 6.16 & 43.5 & 5.067 & 0.461 & 549 & 0.652 \\
\hline-11 & & .40 & 7.94 & 0.59 & 14.00 & 9.2 & 12.85 & 106.24 & 9.55 & 7.39 & 8.27 & 101.6 & 6.467 & 0.323 & .502 & 0.478 \\
\hline & & .13 & 8.58 & 0.61 & 14.00 & 1 & 10.18 & 8.00 & 8.48 & 7.22 & 7.67 & 78.7 & 6.933 & 0.533 & .718 & 0.851 \\
\hline-13 & & & 6.61 & 0. & 0 & & 10.54 & 9.1 & 7.3 & 5.19 & 5.62 & 58. & 5.067 & 0.633 & 730 & 0.733 \\
\hline & & 80 & 5.99 & 0.40 & 17.00 & & 9.33 & 6 & 7.95 & 7.36 & 7.46 & 73.60 & 4.600 & 0.511 & .935 & 0.765 \\
\hline-15 & & .33 & 8.16 & 0.61 & 15.00 & $\overline{6}$ & 9.42 & 69.98 & 7.25 & 6.60 & 7.43 & 66.64 & 6.467 & 0.497 & .607 & 0.741 \\
\hline-16 & & 87 & 7.90 & 0.53 & 16.00 & 2 & 11.48 & 81.38 & 8.98 & 7.03 & 7.09 & 86.41 & 6.467 & 0.269 & .458 & 0.471 \\
\hline & & .53 & 6.53 & 0.48 & 15.0 & 7 & 8.12 & 49.21 & 6.07 & 5.47 & 6.06 & 47.57 & 5.333 & 0.356 & .425 & 0.486 \\
\hline & & .67 & 8.98 & 0.66 & 13.00 & 1 & 10.94 & 103.24 & 9.00 & 8.60 & 9.44 & 100.79 & 7.733 & 0.430 & 0.539 & 0.587 \\
\hline-19 & & 3.07 & 8.18 & 0.63 & 12.00 & 9.6 & 9.02 & 73.36 & 7.74 & 7.08 & 8.13 & 68.47 & 6.933 & 0.533 & 0.560 & 0.677 \\
\hline & & .00 & 9.58 & 0.64 & 13.00 & 1 & 11.32 & 5.97 & 9.79 & 8.48 & 8.48 & 102 & 8.400 & 0.933 & 0.830 & 0.951 \\
\hline & & & 7.02 & 0.49 & & & 8.24 & 50.77 & 6.27 & 5.88 & 6.16 & & 5.733 & 0.410 & .547 & 0.648 \\
\hline & & & 8.70 & 0.65 & & 10 & 10.25 & 85.93 & $\begin{array}{l}7.99 \\
\end{array}$ & 7.45 & 8.38 & & 7.333 & 0.407 & 0.555 & 0.649 \\
\hline & & 15.87 & 8.81 & 0.56 & 9.00 & & 9.36 & 72.09 & 8.59 & 8.15 & 7.70 & & 7.400 & 0.493 & 0.627 & 0.729 \\
\hline & & & 8.72 & 0.64 & & & 9.58 & 78.64 & 8.48 & 7.52 & 8.21 & & 7.467 & 0.533 & .576 & 0.702 \\
\hline & & & 7.76 & 0.55 & & & 8.76 & & 7.00 & 6.43 & 6.89 & & 6.400 & 0.427 & 0.555 & 0.680 \\
\hline & & & 10.29 & 0.75 & & & 10.75 & 107.73 & 9.94 & 9.22 & 10.02 & & 9.067 & 0.824 & 0.750 & 0.905 \\
\hline IVTIRFB-27 & DWRB156 & 15.13 & 9.74 & 0.64 & 16.00 & 11.39 & 10.58 & 92.25 & 9.30 & 8.80 & 8.72 & 99.71 & 8.200 & 0.586 & 0.742 & 0.876 \\
\hline
\end{tabular}


Fig.1 Principal component analysis (PC1 vs PC2) plot of ranks based on measures

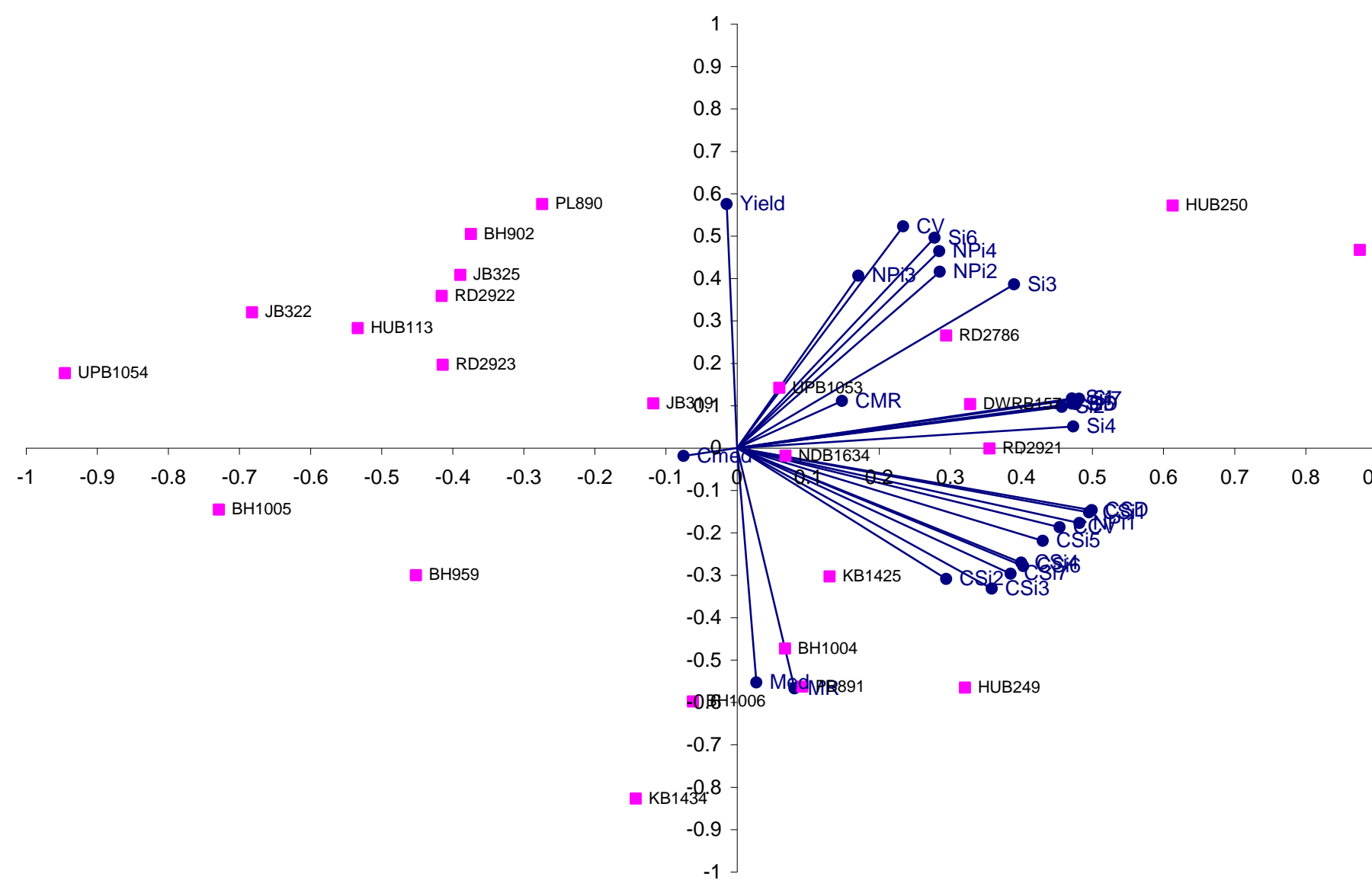

Table.4 Loadings of rank derived from measures for $\mathrm{PC} 1$ and PC2

\begin{tabular}{|l|r|r|}
\hline & PC1 & PC2 \\
\hline Yield & -0.008 & 0.340 \\
\hline MR & 0.042 & -0.335 \\
\hline SD & 0.250 & 0.061 \\
\hline CV & 0.122 & 0.309 \\
\hline Med & 0.014 & -0.327 \\
\hline Si1 & 0.247 & 0.069 \\
\hline Si2 & 0.239 & 0.057 \\
\hline Si3 & 0.204 & 0.228 \\
\hline Si4 & 0.248 & 0.030 \\
\hline Si5 & 0.247 & 0.062 \\
\hline Si6 & 0.146 & 0.293 \\
\hline Si7 & 0.252 & 0.069 \\
\hline CMR & 0.077 & 0.066 \\
\hline CSD & 0.261 & -0.087 \\
\hline CCV & 0.238 & -0.111 \\
\hline Cmed & -0.039 & -0.011 \\
\hline CSi1 & 0.259 & -0.090 \\
\hline CSi2 & 0.154 & -0.183 \\
\hline CSi3 & 0.188 & -0.196 \\
\hline CSi4 & 0.209 & -0.160 \\
\hline CSi5 & 0.225 & -0.129 \\
\hline CSi6 & 0.211 & -0.165 \\
\hline CSi7 & 0.201 & -0.176 \\
\hline NPi1 & 0.252 & -0.105 \\
\hline NPi2 & 0.149 & 0.246 \\
\hline NPi3 & 0.089 & 0.241 \\
\hline NPi4 & 0.149 & 0.275 \\
\hline$\%$ & & \\
Variance & 49.36 & 31.23 \\
\hline & & \\
\hline
\end{tabular}


Table.5 Speraman's rank correlation of yield with non-parametric measures calculated from original and corrected values

\begin{tabular}{|c|c|c|c|c|c|c|c|c|c|c|c|c|c|c|c|c|c|c|c|c|c|c|c|c|c|c|}
\hline & Yield & MR & SD & $\mathrm{CV}$ & Med & $\mathrm{S}_{\mathrm{i}}{ }^{1}$ & $\mathrm{~S}_{\mathrm{i}}{ }^{2}$ & $\mathrm{~S}_{\mathrm{i}}^{3}$ & $\mathrm{~S}_{\mathrm{i}}^{4}$ & $\mathrm{~S}_{\mathrm{i}}^{5}$ & $\mathrm{~S}_{\mathrm{i}}{ }^{6}$ & $\mathrm{~S}_{\mathrm{i}}{ }^{7}$ & CMR & CSD & $\mathrm{CCV}$ & CMed & CSi1 & CSi2 & CSi3 & CSi4 & CSi5 & CSi6 & CSi7 & $\mathrm{NP}_{\mathrm{i}}{ }^{(1)}$ & $\mathrm{NP}_{\mathrm{i}}^{(2)}$ & $\mathrm{NP}_{\mathrm{i}}{ }^{(3)}$ \\
\hline MR & -0.950 & & & & & & & & & & & & & & & & & & & & & & & & & \\
\hline SD & 0.070 & 0.105 & & & & & & & & & & & & & & & & & & & & & & & & \\
\hline CV & 0.817 & -0.729 & 0.553 & & & & & & & & & & & & & & & & & & & & & & & \\
\hline Med & -0.889 & 0.931 & -0.027 & -0.775 & & & & & & & & & & & & & & & & & & & & & & \\
\hline $\mathrm{S}_{\mathrm{i}}{ }^{\mathrm{I}}$ & 0.051 & 0.113 & 0.984 & 0.535 & -0.003 & & & & & & & & & & & & & & & & & & & & & \\
\hline $\mathrm{S}_{\mathrm{i}}^{2}$ & 0.115 & 0.042 & 0.957 & 0.599 & -0.078 & 0.922 & & & & & & & & & & & & & & & & & & & & \\
\hline $\mathrm{S}_{\mathrm{i}}^{3}$ & 0.587 & -0.464 & 0.786 & 0.921 & -0.552 & 0.781 & 0.793 & & & & & & & & & & & & & & & & & & & \\
\hline $\mathrm{S}_{\mathrm{i}}^{4}$ & -0.072 & 0.226 & 0.933 & 0.438 & 0.102 & 0.951 & 0.894 & 0.690 & & & & & & & & & & & & & & & & & & \\
\hline $\mathrm{S}_{\mathrm{i}}^{5}$ & 0.015 & 0.163 & 0.967 & 0.481 & 0.038 & 0.978 & 0.866 & 0.739 & 0.915 & & & & & & & & & & & & & & & & & \\
\hline $\mathrm{S}_{\mathrm{i}}{ }^{6}$ & 0.773 & -0.671 & 0.603 & 0.975 & -0.737 & 0.590 & 0.605 & 0.946 & 0.484 & 0.567 & & & & & & & & & & & & & & & & \\
\hline $\mathrm{S}_{\mathrm{i}}{ }^{7}$ & 0.070 & 0.105 & 1.000 & 0.553 & -0.027 & 0.984 & 0.957 & 0.786 & 0.933 & 0.967 & 0.603 & & & & & & & & & & & & & & & \\
\hline CMR & 0.392 & -0.172 & 0.281 & 0.283 & -0.045 & 0.260 & 0.200 & 0.267 & 0.200 & 0.276 & 0.305 & 0.281 & & & & & & & & & & & & & & \\
\hline CSD & -0.239 & 0.433 & 0.756 & 0.193 & 0.278 & 0.741 & 0.701 & 0.422 & 0.760 & 0.734 & 0.237 & 0.756 & 0.155 & & & & & & & & & & & & & \\
\hline $\mathrm{CCV}$ & -0.353 & 0.496 & 0.672 & 0.097 & 0.319 & 0.665 & 0.653 & 0.331 & 0.710 & 0.654 & 0.128 & 0.672 & -0.172 & 0.916 & & & & & & & & & & & & \\
\hline CMed & 0.002 & 0.111 & -0.017 & -0.182 & 0.358 & -0.005 & -0.009 & -0.141 & 0.013 & -0.015 & -0.187 & -0.017 & 0.572 & -0.126 & -0.246 & & & & & & & & & & & \\
\hline cSil & -0.270 & 0.447 & 0.757 & 0.171 & 0.295 & 0.742 & 0.710 & 0.402 & 0.765 & 0.736 & 0.211 & 0.757 & 0.097 & 0.988 & 0.939 & -0.131 & & & & & & & & & & \\
\hline cSi2 & -0.248 & 0.392 & 0.416 & 0.013 & 0.232 & 0.328 & 0.418 & 0.096 & 0.425 & 0.337 & 0.034 & 0.416 & 0.179 & 0.687 & 0.609 & -0.111 & 0.659 & & & & & & & & & \\
\hline cSi3 & -0.319 & 0.474 & 0.514 & 0.000 & 0.310 & 0.458 & 0.506 & 0.153 & 0.553 & 0.460 & 0.030 & 0.514 & 0.070 & 0.860 & 0.824 & -0.118 & 0.855 & 0.898 & & & & & & & & \\
\hline cSi4 & -0.299 & 0.455 & 0.495 & -0.003 & 0.325 & 0.469 & 0.440 & 0.157 & 0.587 & 0.470 & 0.047 & 0.495 & 0.244 & 0.832 & 0.730 & -0.035 & \begin{tabular}{|l|}
0.800 \\
\end{tabular} & 0.870 & 0.934 & & & & & & & \\
\hline cSi5 & -0.237 & 0.436 & 0.543 & 0.033 & 0.324 & 0.522 & 0.491 & 0.207 & 0.581 & 0.521 & 0.066 & 0.543 & 0.251 & 0.910 & 0.805 & 0.028 & 0.896 & 0.722 & 0.912 & 0.897 & & & & & & \\
\hline cSi6 & -0.308 & 0.469 & 0.535 & 0.004 & 0.329 & 0.514 & 0.509 & 0.182 & 0.585 & 0.511 & 0.026 & 0.535 & 0.038 & 0.904 & 0.894 & \begin{tabular}{|l|}
-0.107 \\
\end{tabular} & 0.910 & 0.716 & 0.938 & 0.869 & 0.959 & & & & & \\
\hline cSi7 & -0.287 & 0.464 & 0.515 & 0.009 & 0.318 & 0.462 & 0.501 & 0.153 & 0.555 & 0.460 & 0.034 & 0.515 & 0.202 & 0.857 & 0.772 & -0.045 & 0.838 & 0.901 & 0.985 & 0.960 & 0.929 & 0.922 & & & & \\
\hline $\mathrm{NP}_{\mathrm{i}}{ }^{(1)}$ & -0.357 & 0.552 & 0.729 & 0.074 & 0.388 & 0.724 & 0.655 & 0.322 & 0.758 & 0.729 & 0.125 & 0.729 & 0.117 & 0.975 & 0.917 & -0.077 & 0.972 & 0.671 & 0.838 & 0.819 & 0.882 & 0.878 & 0.835 & & & \\
\hline $\mathrm{NP}_{\mathrm{i}}{ }^{(2)}$ & 0.733 & -0.692 & 0.518 & 0.900 & -0.831 & 0.503 & 0.518 & 0.855 & 0.405 & 0.469 & 0.906 & 0.518 & 0.106 & 0.247 & 0.203 & -0.357 & 0.224 & 0.044 & 0.083 & 0.060 & 0.110 & 0.105 & 0.063 & 0.154 & & \\
\hline $\mathrm{NP}_{\mathrm{i}}{ }^{(3)}$ & 0.824 & -0.745 & 0.323 & 0.853 & -0.781 & 0.290 & 0.374 & 0.730 & 0.221 & 0.247 & 0.837 & 0.323 & 0.263 & 0.178 & 0.086 & $\mid-0.163$ & 0.132 & 0.126 & 0.143 & 0.133 & 0.181 & 0.143 & 0.153 & 0.023 & 0.849 & \\
\hline $\mathrm{NP}_{\mathrm{i}}{ }^{(4)}$ & 0.827 & -0.730 & 0.488 & 0.949 & -0.776 & 0.477 & 0.531 & 0.869 & 0.389 & 0.419 & 0.924 & 0.488 & 0.286 & 0.247 & 0.153 & \begin{tabular}{|l|}
-0.145 \\
\end{tabular} & 0.223 & 0.052 & 0.103 & 0.089 & 0.170 & \begin{tabular}{|l|}
0.136 \\
\end{tabular} & 0.109 & 0.115 & 0.915 & 0.936 \\
\hline
\end{tabular}

Critical values of Spearman correlation at $5 \%$ and $1 \%$ level of significance (df 25) are 0.398 and 0.510 respectively 
Fig.2 Hierarchical cluistering of feed barley genotypes as per non parametric measures

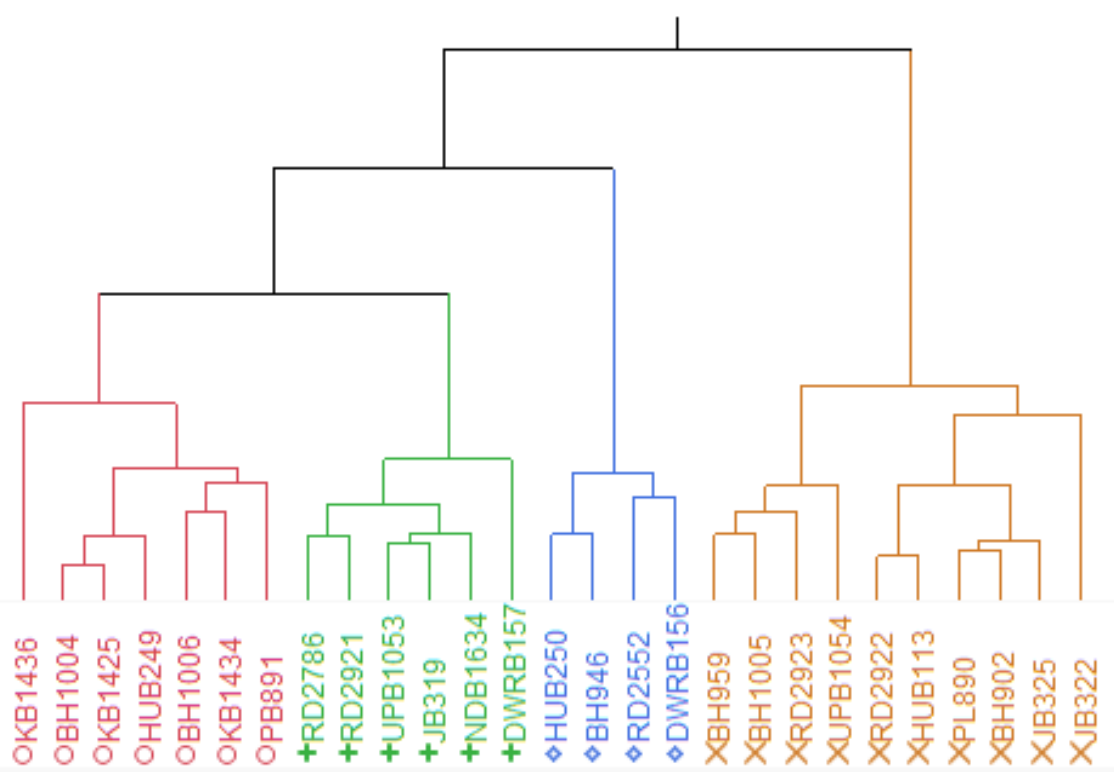

Relationship among nonparametric statistics

Spearman's rank correlations among rank of genotypes as per various non-parametric measures were then calculated (Table 5). According to results of rank correlations there was a highly significant $(\mathrm{p}<0.01)$ positive rank correlation between mean yield with Si3,Si6 NPi(2) NPi(3), NPi(4) and highly significant negative association with MR and CV (Mohammadi et al., 2007). Yield expressed low correlation of inverse relation with CSi1, CSi2, CSi3, CSi4, CSi5, CSi6 and CSi7. MR had significant negative rank correlation with CV, Si6 NPi (2) NPi(3) $\mathrm{NPi}(4)$ whereas significant positive with CSi1,CSi2,CSi3,CSi4,CSi5,CSi6 and CSi7 (Mahtabi et al., 2013). SD had a highly significant positive with most of the measures either based on original or corrected values. Sil showed highly significant positive rank correlation with $\mathrm{Si} 2, \mathrm{Si} 3, \mathrm{Si} 4 \mathrm{Si}$,Si6,Si7 CSi1,CSi5 CSi6, NPi(1) and significant Positive CSi3, CSi6, NPi (2) and NPi(4) (Mohammadi and Ahmed, 2008). Significant positive association among $\mathrm{Sis}, \mathrm{Si} 3$ showed significant correlation with $\mathrm{NPi}(\mathrm{s})$.

$\mathrm{Si} 4$ and. Si3 maintained same type of relationship with other measures. Similar behavior expressed by $\mathrm{Si} 7$ to show positive relationship. CSD showed significant positive correlation with CSis, and with very low positive interaction with $\mathrm{NPi}(\mathrm{s})$. CSi1 had positive significant relationship and very low with NPi(s). More over CSis were positively associated among themselves. NPi(2) expressed significant positive rank correlation with $\mathrm{NPi}(3)$ and $\mathrm{NPi}(4)$ (Mortazavian and Azizinia, 2014).

\section{Biplot analysis of non-parametric measures}

Principal component (PC) analysis based on the rank correlation matrix generated by non para-metric measures was performed understand relationships if any among these measures. Table 4 shows the loading of the first two PCA of ranks of non-parametric measures as two first PCs (PC1 and PC2) explained $80.6 \%$ (49.36 and $31.23 \%$ by $\mathrm{PC} 1$ 
and PC2, respectively) of the total variance. Better visualization of relationships among the different measures and yield (Y) displayed graphically by biplot (Dehghani et al., 2009). In this plot, the PC1 axis mainly distinguished mean yield besides the measures of $\mathrm{CV}, \mathrm{MR}$ and (6) is from the other measures.

Thus, the first principal component separated the measures into two groups according to the two stability concepts (biological and agronomic concept of stability). The second PC separated the nonparametric measures of phenotypic stability into two groups according to the yield and stability (Fig. 1). The original data-based nonparametric measures showed close correlation with $\mathrm{CV}$, $\mathrm{Si} 3 \mathrm{Si} 6$ and no relation with CMR, Si4, $\mathrm{Si} 7$ as vectors corresponding to these measures expressed right angle with vector of yield (Mortazavian and Azizinia, 2014). Genotypes HUB250, RD2786, DWRB156, UPB1053 and DWRB157 clustered with measures based on original yield values. Corrected data-based nonparametric measures were closely related among themselves and clustered together.

Yield showed nearly straight line angle with vectors of MR and Median. These measures favored HUB249, KB1425, BH1004 and PL891.

\section{Acknowledgement}

The multi-environment trials of barley genotypes were performed within the AICWandBIP project at centers across the country. Authors are grateful to all the staff of testing centers for their hard work to carry out the field evaluation and data recording.

\section{References}

Berteroa, H.D., de la Vegab, A.J., Correaa, G., Jacobsenc, S.E., Mujica, A. 2004. Genotype and genotype-by-environment interaction effects for grain yield and grain size of quinoa (Chenopodium quinoa Willd.) as revealed by pattern analysis of international multienvironment trials. Field Crops Res., 89: 299-318.

Dehghani, H. 2008. Estimating yield stability by nonparametric stability analysis in maize (Zea mays L.). Plant Breed Seed Sci., 58: 61-77.

Dehghani, H., Sabaghnia, N., Moghaddam, M. 2009. Interpretaרtion of genotypeby-environment interaction for late maize hybrids' grain yield using a biplot method. Turk. J. Agric. For., 33: 139148.

Ebadi-Segherloo, A., Sabaghpour, S.H., Dehghani, H., Kamrani, M. 2008. Nonparametric measures of phenotypic stability in chickpea genotypes (Cicer arietinum L.). Euphytica, 2: 221-229.

Farshadfar, E., Mahmudi N. and Sheibanirad, A. 2014. Nonparametric methods for interpreting genotype $\times$ environment interaction in bread wheat genotypes. $J$. Bio. Env. Sci., 4: 55-62.

Huehn, M.1990a. Non-parametric measures of phenotypic sta $\neg$ bility. Part 1: Theory. Euphytica, 47:189-194.

Huehn, M.1990b. Non-parametric measures of phenotypic stability: Part 2. Application. Euphytica, 47: 195-201.

Hussein, M.A., Bjornstad, A., Aastveit, A.H. 2000. SASG $\times$ ES $\neg$ TAB: A SAS program for computing genotype 3 environment stability statistics. Agron. J., 92: 454-459.

Karimizadeh, R., Mohammadi, M., Sabaghnia, N. and Shefazadeh, M.K. 2012. Using Huehn's nonparametric stability statistics to investigate genotype $\times$ environment interaction. Not. Bot. Horti. Agrobo., 40: 195-200.

Karimzadeh, R., Mohammadi M., Sabaghnia N., Shefazadeh M.K., Hosseinpour, T. and Armion, M. 2013. Exploring of 
genotype by environment interaction by nonparametric stability procedures. Natura montenegrina, 12: 181-204.

Khalili, M., and Pour-Aboughadareh, A. 2016. Parametric and non-parametric measures for evaluating yield stability and adaptability in barley doubled haploid lines. J. Agri. Sci. Tech., 18: 789-803

Liu, Y.J., Duan, C., Tian, M.L., Hu, E.L., Huang, Y.B. 2010. Yield stability of maize hybrids evaluated in maize regional trials in southwestern China using nonparametric methods. Agric. Sci. China, 9: 1413-1422.

$\mathrm{Lu}$, H.Y. 1995. PC-SAS program for Estimation Huehn's non parametric stability statistics. Agron. J., 87: 888891.

Mahtabi, E., Farshadfar, E. and Jowkar, M.M. 2013. Non parametric estimation of phenotypic stability in Chickpea (Cicer arietinum L.). Int. J. Agri. Crop Sci., 5: 888-895.

Mohammadi, R., Abdulahi, A., Haghparast, R., Armion, M. 2007. Interpreting genotype $\mathrm{x}$ environment interactions for durum wheat grain yields using nonparametric methods. Euphytica, 157: 239-251.

Mohammadi, R. and Ahmed, A. 2008. Comparison of parametric and non- parametric methods for selecting stable and adapted durum wheat genotypes in variable environments, Euphytica, 159: 419-432.

Mortazavian, S.M.M. and Azizinia, S. 2014. Nonparametric stability analysis in multi-environment trial of canola. Turkish J. Field Crops, 19(1): 108-117.

Sabaghnia, N., Dehghani, H., Sbaghpour, S.H. 2006. Nonparametric methods for interpreting genotype $\times$ environment interaction of lentil genotypes. Crop Sci., 46: 1100-1106.

Thennarasu, K. 1995. On certain nonparametric procedures for studying genotype $\times$ environment interactions and yield stability. PhD. Thesis. P.J. School, IARI, New Delhi, India.

Ward J.H. 1963. Hierarchical grouping to optimize an objective function. $J$. Am. Stat. Assoc., 58: 236-224.

Yau, S.K., Hamblin, J. 1994. Relative yield as a measure of entry performance in variable environments. Crop Sci., 34: 813-817.

Zali, H., Farshadfar, E. and Sabaghpour, H. 2011. Non-parametric analysis of phenotypic stability in chickpea (Cicer arietinum L.) genotypes in Iran. Crop Breeding J., 1: 85-96.

\section{How to cite this article:}

Ajay Verma, J. Singh, V. Kumar, A.S. Kharab and Singh, G.P. 2017. Non Parametric Analysis in Multi Environmental Trials of Feed Barley Genotypes. Int.J.Curr.Microbiol.App.Sci. 6(6): 1201-1210. doi: https://doi.org/10.20546/ijcmas.2017.606.139 